\title{
T- filters based dual-control dimmable electronic ballast
}

\author{
Huadong Wang ${ }^{1, \text { a }}, \mathrm{Xu} \mathrm{Cai}^{2, \mathrm{~b}}$,Lili Liu ${ }^{3, \mathrm{c}^{*}}$ \\ 1 Electronic Information and Electrical Engineering, Electrical Engineering, Shanghai Jiao Tong \\ University, Shanghai, China \\ 2 Electronic Information and Electrical Engineering, Electrical Engineering, Shanghai Jiao Tong \\ University, Shanghai, China \\ 3 Jiangsu Key Laboratory of Large Engineering Equipment Detection and Control, Xuzhou Institute \\ of Technology, Xuzhou, Jiangsu, China \\ ahuadong.wang@shsdec.com, bxucai@sjtu.edu.cn, dxwlll@sina.com
}

Keyword: HID; Acoustic resonator;T-type filter; Dimmer; Dual control

Abstract: This paper describes a method for the analysis and design of dual control dimming UHF electronic ballasts. It includes a PFC converter circuit and a T-type inverter circuit, the ballast operating at the highest point above the acoustic resonance frequency can effectively avoid the high-intensity discharge (HID) lamps acoustic resonance problems. T-type filter can achieve a very narrow depth range of dimming frequency, while achieving low lamp current crest factor, keeping the lamp current a perfect sine wave. Dimming with dual control, adjusting the duty cycle of the two frequencies to change the lamp power can reduce the EMI of FM dimming. Its effectiveness has been verified by experiments.

\section{Introduction}

HID lights acoustic resonant frequency have a relationship with the lamp geometry, the lamp gas parameters, is generally considered the acoustic resonance occurs at a specific frequency range (several tens of $\mathrm{KHz}$ ). Acoustic Resonance can cause the light arc instability and the fluctuation of output power or even burst the lamp. Therefore, how to avoid the occurrence of HID lights Acoustic Resonance, is a major topic of the research on electronic ballast. There are the commonly used methods: DC, low frequency square-wave, sweep and UHF work. The smaller values of high frequency magnetic components, smaller in size, but the higher costs of the drive chip, but also high-frequency magnetic material and higher costs, general elections at $300 \mathrm{~K} \sim 400 \mathrm{~K}$ frequencies, can effectively overcome the acoustic resonance, cost and size are minimal. The higher the frequency of the magnetic component, the value is smaller, the size is smaller, but the higher the cost driver chip, but also need a high-frequency magnetic material costs, the general election in the frequency of $300 \mathrm{~K} \sim 400 \mathrm{~K}$, can effectively overcome the acoustic resonance, and the cost is the smallest. Loss is greater with the higher frequency switch, a reasonable choice of topology and parameters designed to make the switch work in the soft switch, to ensure high efficiency.

Ballast dimming is also a direction of research. Commonly used methods as follows:

1. Adjustable light [1]: PFC usually used BOOST circuit, voltage is limited and the dimming range is limited.

2. Modulation the duty cycle dimming [2]: full-bridge inverter circuit, the control method is complicated and costly. Half-bridge inverter circuit, lamp crest factor is higher, shorten the life of the lamp.

3. FM dimming [3]: series and parallel inverter circuit commonly used to achieve deep dimming 
frequency changes, the switch over a wide frequency range are difficult to achieve soft switching, the article [4] presented deep dimming by T-filter narrower frequency range. However, the continuous frequency changes make the difficult to the EMI filter design.

This paper based on the dual control dimming method based on t-type UHF electronic ballast dimming: Lamps operating on two frequency, achieve dimming by changing the duty ratio of the two frequencies. If the two frequencies are fast enough there will be no Flash. For there are only two operating frequencies, the EMI filter design is easy. T-type filter make little difference between the two frequencies, to ensure that the switch is easy to achieve soft switching at two frequencies point, ensuring high efficiency.

\section{Working principle}

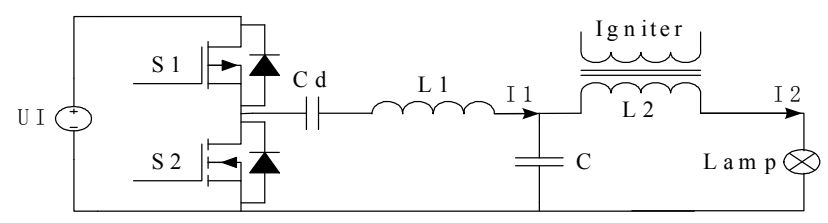

Figure 1 schematic diagram of ballast

Ballast principle is shown in Figure 1, consists of half-bridge Inverter circuit and t-type filter, Cd is blocking capacitor, L2 provide the ignition transformer with high ignition voltage (3KV-4KV), steady-state operation when the secondary side is $\mathrm{T}$ type filter inductor L2.This paper Using parasitic capacitance and parasitic MOSFET body diode to achieve switch ZVS, no additional soft-switching circuit, with low cost efficiency. HID lamp is approximately equivalent to a resistance $\mathrm{R}$ when operations at a steady-state, T-type filter voltage transmission characteristics:

$$
H_{T}(j \omega)=\frac{R}{-j \omega^{3} L_{1} L_{2} C-\omega^{2} L_{1} C R+j \omega\left(L_{1}+L_{2}\right)+R}
$$

Figure $2|\mathrm{H}|$-f curve $(|\mathrm{H}|$ is the voltage transfer ratio for values). Figure (1), Figure 2 shows t-filters can be accomplished dimming within a very narrow frequency range. Select of the T-type filter parameters determine whether the circuit can work properly and the switch can achieve soft switching, a reasonable choice of L1, L2, and C values, can make T-type filter lamp ignition, the lamp lights up and working properly have shown sensibility working condition, to ensure the realization of soft-switching.

State approximation is open when the ignition, can be obtained:

$$
\begin{gathered}
Z_{o}(j \omega)=\frac{1-L_{1} C \omega^{2}}{j C \omega} \\
\angle Z_{o}(j \omega)=\frac{\pi}{2}
\end{gathered}
$$

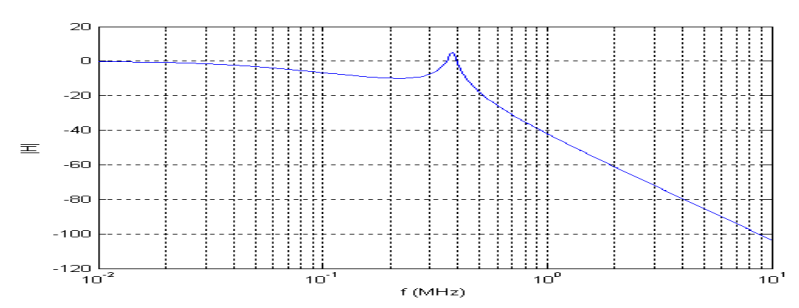

Figure 2 voltage transfer ratio

The start can be approximately the short-circuited state, can be obtained:

$$
Z_{s}(j \omega)=\frac{j \omega\left(L_{1}+L_{2}-L_{1} L_{2} C \omega^{2}\right)}{1-L_{2} C \omega^{2}}
$$




$$
\begin{gathered}
\angle Z_{s}(j \omega)=\frac{\pi}{2} \\
f_{1}=\frac{1}{2 \pi \sqrt{\frac{L_{1} L_{2}}{L_{1}+L_{2}} C}} \\
I_{1}=\frac{\left|1-L_{2} C \omega^{2}\right|}{\left(L_{1}+L_{2}-L_{1} L_{2} \omega^{2}\right) \omega} U_{I 1} \\
I_{2}=\frac{1}{\left(L_{1}+L_{2}-L_{1} L_{2} \omega^{2}\right) \omega} U_{I 1}
\end{gathered}
$$

At the steady state, the lamp is equivalent to the resistor $\mathrm{R}(\mathrm{R}=\mathrm{U} 2$ / P), can be obtained:

$$
\begin{gathered}
\left|H\left(j \omega_{0}\right)\right|=\frac{1}{\sqrt{\left(1-\omega_{0}{ }^{2} L_{1} C\right)^{2}+\frac{\omega_{0}{ }^{2}}{R^{2}}\left(L_{1}+L_{2}-L_{1} L_{2} \omega_{0}{ }^{2}\right)^{2}}}=H_{0}=\frac{V_{o m}}{\frac{2}{\pi} V_{I}} \\
\left|Z\left(j \omega_{0}\right)\right|=\frac{R}{H_{0} \sqrt{\left(1-L_{2} C \omega_{0}{ }^{2}\right)^{2}+\left(R C \omega_{0}\right)^{2}}} \\
\angle Z\left(j \omega_{0}\right)=\arctan \frac{\omega_{0}\left(L_{1}+L_{2}-L_{1} L_{2} C \omega_{0}{ }^{2}\right)}{R\left(1-L_{1} C \omega_{0}{ }^{2}\right)}-\arctan \frac{R C \omega_{0}}{1-L_{2} C \omega_{0}{ }^{2}}
\end{gathered}
$$

Switching frequency f0 $>\mathrm{fl}$ to meet dimming characteristic. Metal halide lamp ignition current should be more than twice the rated current, we can learned by (8) that the L2 should be larger; In order to reduce the loss of the switch, current should be as small as possible, we can learned by (7) that the L2 should be larger, therefore, the L2 should consider a compromise, a reasonable choice of L2 can make the circuit can work properly. Soft switch realization: from (3) (5) we can see, we can achieve soft switching at ignition and start. Steady operation without considering the impact of parasitic capacitance:

$$
\begin{gathered}
\left|\overline{i_{1}}\right|=\frac{\sqrt{2} U_{I 1}}{\left|Z\left(j \omega_{0}\right)\right|} \sin \left(\angle\left(Z\left(j \omega_{0}\right)\right)\right) \\
=\frac{H_{0}^{2}}{R^{2}} \sqrt{2} U_{I 1}\left[\omega_{0}\left(L_{1}+L_{2}-L_{1} L_{2} \omega_{0}{ }^{2}\right)\left(1-L_{2} C \omega_{0}{ }^{2}\right)-R^{2}\left(1-L_{1} C \omega_{0}{ }^{2}\right) C \omega_{0}\right] \\
T_{1}=\frac{2 C_{S} U_{I 1}}{\left|\overline{i_{1}}\right|}
\end{gathered}
$$

To sum up: T-resonant circuit parameters should satisfy (14), in order to meet the deep dimming, ignition, start-up and during the entire dimming soft switching characteristics.

$$
\left\{\begin{array}{c}
f_{0}>\frac{1}{2 \pi \sqrt{\frac{L_{1} L_{2}}{L_{1}+L_{2}}}} \\
\frac{1}{\sqrt{\left(1-\omega_{0}{ }^{2} L_{1} C\right)^{2}+\frac{\omega_{0}^{2}}{R^{2}}\left(L_{1}+L_{2}-L_{1} L_{2} \omega_{0}{ }^{2}\right)^{2}}}=H_{0}=\frac{V_{o m}}{\frac{2}{\pi} V_{I}} \\
\pi(1-2 D)-\omega_{0} T_{1}<\angle Z\left(j \omega_{0}\right) \\
I_{2}=\frac{1}{\left(L_{1}+L_{2}-L_{1} L_{2} \omega^{2}\right) \omega} U_{I 1}>=2 I_{\text {rating }} \\
I_{1}=\frac{\left|1-L_{2} C \omega^{2}\right|}{\left(L_{1}+L_{2}-L_{1} L_{2} \omega^{2}\right) \omega} U_{I 1}<I_{\text {MAXswich }}
\end{array}\right.
$$

\section{Dual control}

FM dimming ballasts, fundamental and harmonic frequencies are adjusted with the change in light output varies; create difficulties to the EMI filter design [5]. This paper use the dual control to 
solve this problem, two fundamental switching frequencies is control by a modulation frequency. With only two harmonic frequencies exist, EMI filter design easy. Since the control of modulation frequency so that the switching frequency of the two harmonic power density is very small, which in turn makes EMI filtering criteria easily met.

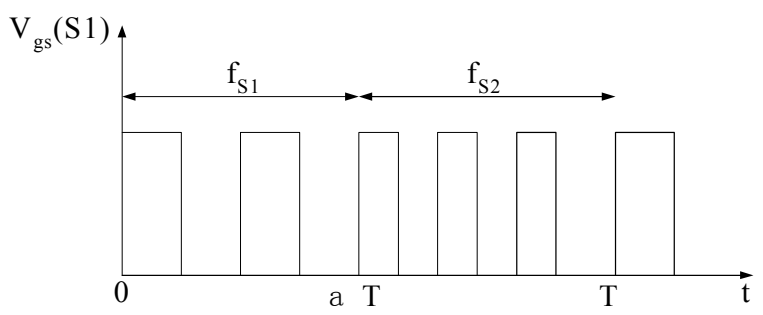

Figure 3 dual control switch drive waveform

Dual control principle: changing switching frequency fS1 and fS2 duration in one cycle of t1 and $\mathrm{t} 2$, varying the ratio of the rated power and minimum power, to achieve the purpose of adjusting the power output. In the dimming stage the lamp operates in two alternating frequencies, and $\mathrm{t} 1$ and $\mathrm{t} 2$ are continuous times, power control is achieved by changing the length of $\mathrm{t} 1$ and $\mathrm{t} 2$. Driving waveform shown in Figure 3, $\mathrm{t} 1$ and $\mathrm{t} 2$ and the relationship between the modulation duty ratio $\alpha$ of: $\mathrm{t} 1=\alpha \times \mathrm{T}, \mathrm{t} 2=(1-\alpha) \times \mathrm{T}$, where $\mathrm{T}$ is the modulation period. Modulation duty cycle $\alpha=0$, ballast completely work at the highest frequency point, minimum output power; $\alpha=1$, ballasts fully operational point of the lowest frequency, maximum output power. When $\alpha$ is between 0 and 1 , the output power ranging between minimum and maximum, the output power increases with increasing $\alpha$. So the changes in $\alpha$ can achieve dimming. In dual control, two basic switching frequency fS1, fS2 and the choice of modulation frequency is very important for EMI characteristics of the circuit. FS1 and fS2 should be as close as possible, fS1 and f ratio should be between 10 to 100 [6]

\section{Design Considerations}

Parametric design method based on the second part of this article, there is $150 \mathrm{~W}$ metal halide lamp design parameters: $\mathrm{fS} 1=400 \mathrm{KHz}, \mathrm{fS} 2=500 \mathrm{KHz}, \mathrm{f}=10 \mathrm{KHz}, \mathrm{BOOST}$ output voltage is $380 \mathrm{~V}$, lamp's rated power $\mathrm{P}=150 \mathrm{~W}$, rated voltage $\mathrm{U} 0=100 \mathrm{~V}$, the equivalent resistance $\mathrm{R}=67 \Omega$, the duty cycle $\mathrm{D}=3 / 8$, the inductor $\mathrm{L} 1=80 \mathrm{uH}, \mathrm{L} 2=145 \mathrm{uH}$, capacitance $\mathrm{C}=3.4 \mathrm{nF}, \mathrm{Cd}=1 \mathrm{uF}$.

\section{The test results}

Figure 4 is a saber simulation waveform, modulation ratio $\alpha=0.5$, the modulation frequency $\mathrm{f}=$ $10 \mathrm{KHz}$. (A) $400 \mathrm{KHz}$ lamp voltage waveform; (b) $500 \mathrm{KHz}$ lamp voltage waveform; (c) Voltage waveform during the operation of the lamp; (d) The switch current flourier analysis graphics, fundamental frequency $100 \mathrm{KHz}$, from the figure, the highest point at $500 \mathrm{KHz}$, no need to consider the working frequencies, the switching current at $1 \mathrm{MHz}$ EMI, needs to be removed, Other frequency amplitude is very small, the design of EMI filter only to remove the interference of $1 \mathrm{MHz}$ frequency. Thus, the dual-dimming makes EMI filter easy to design.

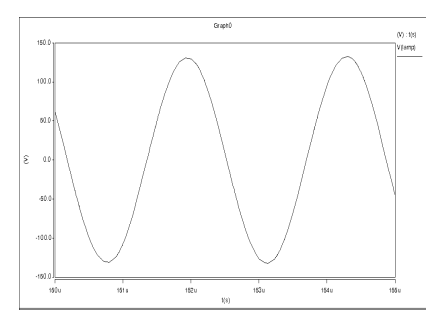

(a) $400 \mathrm{KHz}$ lamp voltage waveform

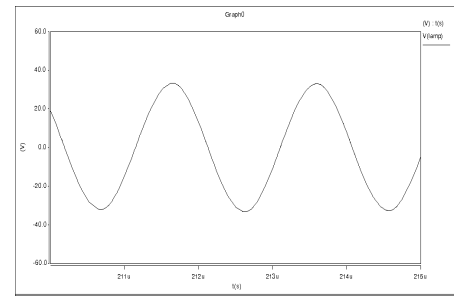

(b) $500 \mathrm{KHz}$ the lamp voltage waveform 


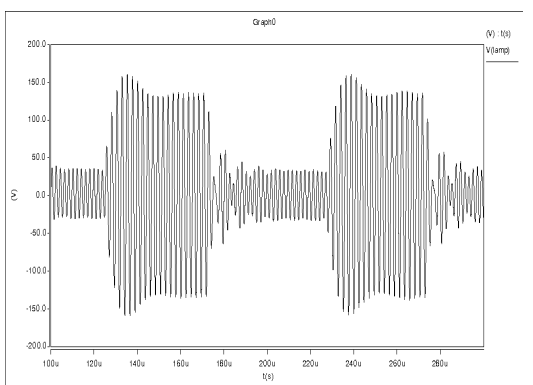

(c)The lamp voltage waveform during operation

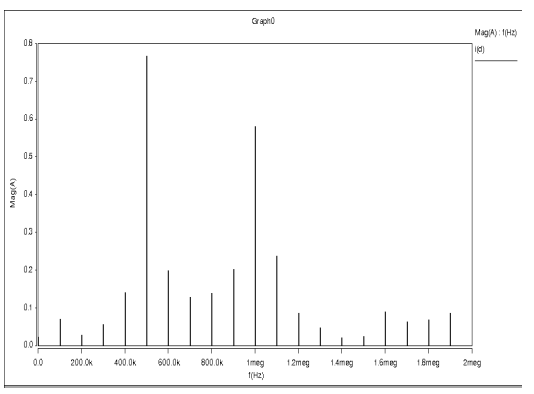

(d) Switch current Fourier synthesis graphical

Figure4 double-frequency control simulation waveforms

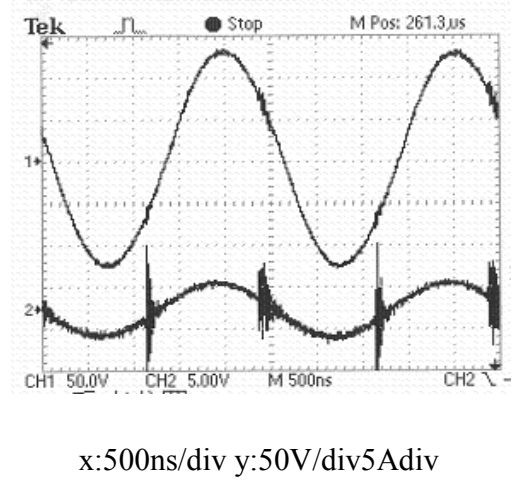

CH1 lamp voltage $\mathrm{CH} 2$ lamp current

(a) $400.7 \mathrm{KHz}$ lamp voltage current waveform

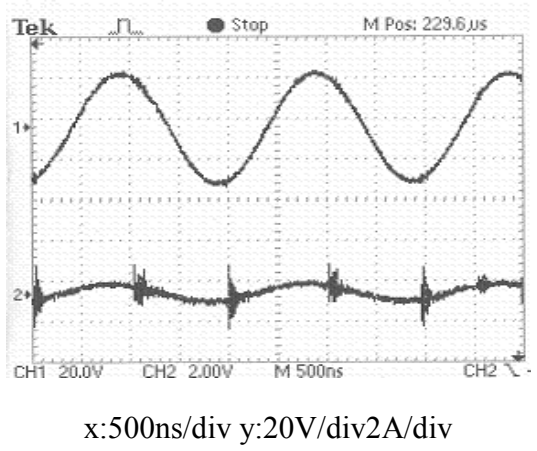

CH1 lamp voltage $\mathrm{CH} 2$ lamp current

(b) $505.1 \mathrm{KHz}$ lamp voltage current waveform

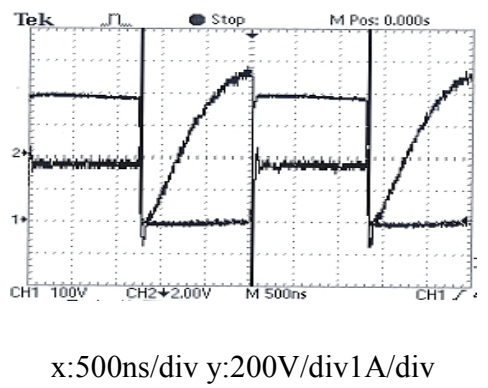

(c) $400.7 \mathrm{KHz}$ Switch tube voltage current waveform 


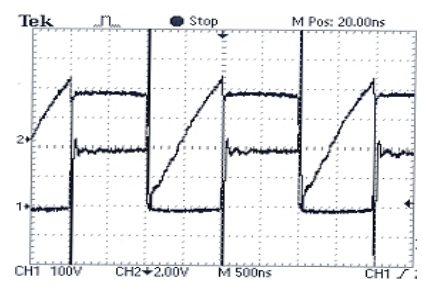

$\mathrm{x}: 500 \mathrm{~ns} / \mathrm{div} \mathrm{y}: 200 \mathrm{~V} / \operatorname{div} 1 \mathrm{~A} /$

(d) $505.1 \mathrm{KHz}$ Switch tube voltage current waveform

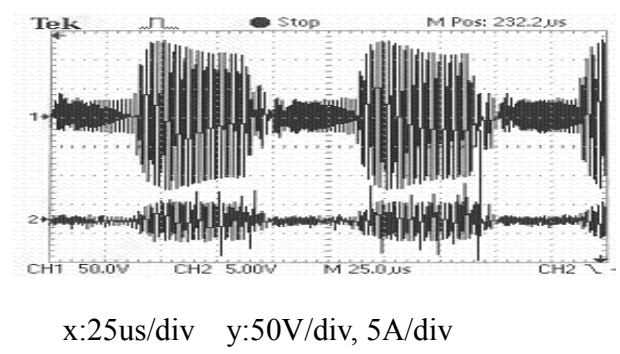

(e) lamp voltage current waveform $\alpha=0.5$

Figure5 Experimental waveforms of lamp voltage and lamp current

Figure5 shows the experimental waveforms (The high frequency component in the lamp current waveform is due to cascade $1 \Omega$ sampling resistance in the measurement) (a) $400.7 \mathrm{KHz}$ lamp voltage current waveform; (b) $505.1 \mathrm{KHz}$ lamp voltage current waveform;(c) $400.7 \mathrm{KHz}$ Switch tube voltage current waveform;(d) $505.1 \mathrm{KHz}$ Switch tube voltage current waveform;(e) When modulation ratio $\alpha=0.5$, the lamp voltage current waveform. From the experimental waveforms: lamp voltage current

Waveform is a perfect sine wave, crest factor is low; Switch tube at two frequencies both to achieve soft switching, switch tube advantages of low loss and efficient. There were no acoustic resonance and stroboscopic throughout the experiment. So double-frequency control is a good method of dimming control

Figure6 is the experimental data of dual frequency dimming's dimming range: dimming range is wide, a linear relationship between modulation and input power.

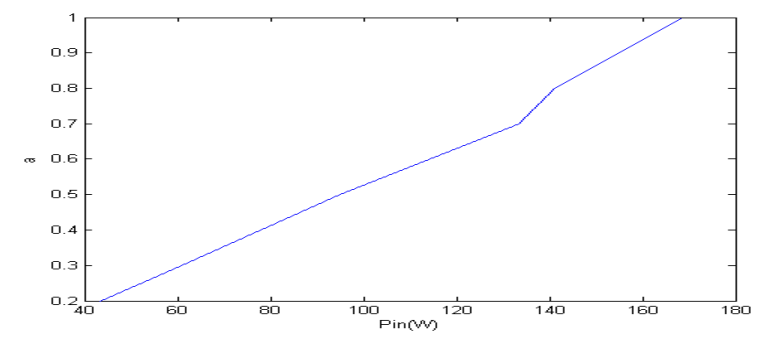

Figure 6 the curve of modulation ratio relative to the input power

\section{Conclusion}

This article presents a novel UHF electronic ballast with dimming feature based on t-type filter, it also describes the operating concept and design method. When the operating frequency exceeds $400 \mathrm{KHz}$, Silent resonance occurred and the lamp voltage current waveform is a perfect sine wave. Deep dimming within a very narrow frequency range can be achieved by t-type filter. There are only two operating frequencies in the dual frequency control, frequency modulation reduces the two frequencies' harmonic power density. Switch at two frequencies both to achieve soft switching, switch tube advantages of low loss and efficient. 


\section{References:}

[1]Van Tichelen, P. Weyen. D. Geeens, R. Lodeweyckx, J. Heremans. G. "A novel dimmable electronic ballast for street lighting with HPS lamps", IEEE IAS'00, 2000.pp.3419-3422

[2]Adams,J. Ribarich, T.J. Ribarich,J.J. "A new control IC for dimmable high-frequency electronic ballast”, IEEE APEC'99, 1999 pp.713-719

[3]Da Silveira Cavalcante, F. Brbi, I. "A new dimmable 70W electronic ballast for high pressure sodium lamps”, IEEE IAS’02, 2002.pp.1856-1862

[4]Yifeng Jiang, Min Chen, Zhaoming Qian “A Novel Dimmable Electronic Ballast With Very High Frequency",IEEE IAS'05, 2005, pp.1954-1957

[5] Y.F.Zhang, L.Yang, C.Q.Lee, "EMI reduction of power supply by bi-frequency modulation", IEEE APEC'94, 1994, pp.601-607

[6] Wu T-F, Yu T-H, "An Electronic Dimming Ballast with Bifrequency and Fuzzy Logic Control." IEEE Trans. on Industry Application, 2000,36(50): 1308-1317 